\title{
The effect of environmental enrichment on hippocampus gene expression and blood corticosterone concentration in Hatano rats
}

\author{
Airi Nakayama ${ }^{1}$, Hasuka Okawa ${ }^{1}$, Hikaru Asano ${ }^{1}$, Hideyo Tokuoka ${ }^{1}$, Meihua Zheng ${ }^{3}$, Shaoxia Pu ${ }^{3}$, \\ Gen Watanabe ${ }^{3,4}$, Ryo Ohta ${ }^{2}$, Maiko Kawaguchi ${ }^{1}$ \\ ${ }^{I}$ Meiji University, Japan, ${ }^{2}$ Hatano Research Institute Food and Drug Safety Center, Japan, ${ }^{3}$ Gifu University, Japan, \\ ${ }^{4}$ Tokyo University of Agriculture and Technology, Japan
}

Environmental enrichment, such as living in a spacious environment and having various toys, will enhances animals' quality of life. In particular, early environmental enrichment exerts a strong biological effect. The purpose of environmental enrichment is to bring out natural behavioral and endocrinological reactions, but it does not always have a good impact on all animals. To explore the effect of environmental enrichment on animals with different characteristics, we studied Hatano high-avoidance (HAA) and low-avoidance (LAA) rats. These rats were originally selected from Sprague-Dawley (SD) rats for high and low active avoidance learning in shuttle box tests, and so have different emotional behavior. Last year, we reported that there were differences in the effect of environmental enrichment on emotional behavior in HAA and LAA rats. In brief, LAA reared in an enriched environment showed lower anxiety-like behavior than LAA reared in a standard environment, but there was no similar enriched environment effect on HAA. Therefore, now we have analyzed gene expression levels in the hippocampus and blood corticosterone concentrations, to examine the factors that caused the previously reported changes in emotional behavior. Male Hatano rats and SD rats were reared in an enriched environment (EE) or standard environment (SE) from 4 weeks old, and were decapitated at 20 weeks of age to collect hippocampus and blood samples. Expression levels of Brain-derived neurotrophic factor (BDNF) genes, Tyrosine receptor kinase $\mathrm{B}(\mathrm{TrKB})$ and Glucocorticoid receptor $(\mathrm{GR})$ were determined by real-time PCR, and the plasma corticosterone level was measured by radioimmunoassay. EE induced more BDNF exon3, 4 and GR expression in HAA and LAA rats. EE also induced greater expression of BDNF exon1 and TrKB in LAA rats. But, there was no difference between SE and EE for BDNF exon3, TrKB, or GR expression in SD rats. Futhermore, there was no effect of EE on blood corticosterone concentration in SD, HAA, or LAA rats.

These results show that TrKB may be involved in reducing anxiety-like behavior. This work was supported by Research Project Grant (A), Institute of Science and Technology, Meiji University. 